\title{
Neck Circumference and Incidence of Type 2 Diabetes in Chinese Elderly Individuals: A Community-Based Cohort Study
}

\author{
Qun Yan ${ }^{a}$ Dongmei Sun ${ }^{b} \quad X$ Li $^{a} \quad$ QingHu Zheng $^{b}$ HaiNing Long $^{b}$ Bo Feng ${ }^{a}$ \\ a Department of Endocrinology, Shanghai East Hospital, Tongji University School of Medicine, Shanghai, China; \\ ${ }^{b}$ Shanghai Puxing Community Health-Care Center, Shanghai, China
}

\section{Keywords}

Neck circumference - Type 2 diabetes mellitus - Waist circumference

\begin{abstract}
Introduction: This study aimed to investigate whether neck circumference (NC) was associated with the incidence of type 2 diabetes in Chinese elderly individuals. Methods: A community-based cohort study was conducted on elderly inhabitants in Shanghai with a mean age of $71.0 \pm 5.8$ years $(n=2,646)$. Binary logistic regression analysis was performed to evaluate the association between NC and the prevalence of type 2 diabetes, while a Cox regression model was used to determine the association between NC and the incidence of type 2 diabetes after a follow-up of 2 years. Results: Logistic regression analysis showed that a larger NC was significantly associated with an increased risk for type 2 diabetes in men (odds ratio [OR] 1.18, 95\% confidence interval [Cl] 1.07-1.31; $p=0.001$ ) and women (OR 1.25, 95\% Cl 1.13-1.38; $p<0.001$ ). Cox regression analysis revealed that NC was independently associated with the incidence of type 2 diabetes in both men (hazard ratio $[\mathrm{HR}] 1.14,95 \% \mathrm{Cl} 1.05-1.23 ; p=0.002$ ) and women (HR 1.18, 95\% Cl 1.10-1.27; $p<0.001)$. Conclusions:
\end{abstract}

karger@karger.com www.karger.com/ofa

Karger $\stackrel{\text { ' }}{5}$
(C) 2021 The Author(s)

Published by S. Karger AG, Basel

This article is licensed under the Creative Commons AttributionNonCommercial-NoDerivatives 4.0 International License (CC BYNC-ND) (http://www.karger.com/Services/OpenAccessLicense) Usage and distribution for commercial purposes as well as any distribution of modified material requires written permission.
A larger NC was associated with a higher risk of developing type 2 diabetes in Chinese elderly individuals. However, studies with larger sample sizes and longer follow-up durations are needed to definitively determine the relationship between $\mathrm{NC}$ and the risk of developing type 2 diabetes.

(c) 2021 The Author(s)

Published by S. Karger AG, Basel

\section{Introduction}

As early as 1995, studies reported that neck circumference (NC), which can be used as an indicator of upperbody subcutaneous adipose tissue, is related to cardiovascular risk factors in severely obese men and women [1]. A series of reports was published on the correlation between NC and obesity [2-9]. Israeli scholars found that NC was a simple and efficient measure of overweight and obesity in adults [2]. A recent meta-analysis of studies from the USA, Turkey, Greece, Brazil, Egypt, and other countries showed that $\mathrm{NC}$ has an area under the curve of 0.871 for identifying overweight and obesity in chil-

Qun Yan and DongMei Sun contributed equally to this work.
Correspondence to:

Bo Feng, fengbodfyy@tongji.edu.cn 
dren and adolescents, with a sensitivity and specificity of 0.78 and 0.75 , respectively [10]. Furthermore, NC was found to be significantly correlated with visceral adipose tissue and carotid intima media thickness in the Chinese population $[11,12]$. Our previous study also identified $\mathrm{NC}$ as a risk factor for obesity and metabolic syndrome, independent of waist circumference (WC), in elderly individuals residing in eastern China [13]. These findings all suggest there is a correlation between $\mathrm{NC}$ and body mass index (BMI), central obesity, and metabolic syndrome.

Although associations between NC and obesity and metabolic syndrome have been well established, research into the relationship between NC and the risk of developing type 2 diabetes, especially in longitudinal settings, has been insufficient [14-16]. This longitudinal follow-up study aimed to evaluate whether $\mathrm{NC}$ is associated with the risk of developing type 2 diabetes in a natural elderly population in eastern China.

\section{Materials and Methods}

\section{Population}

Data were obtained from a retrospective, community-based, epidemiological study on type 2 diabetes involving the elderly population $(n=2,646)$. Study participants were local residents aged $\geq 65$ years from 14 urban communities in Shanghai Pudong New District. The survey was conducted from April to August 2014 and the follow-up was conducted in 2016. Overall, a total of 5,282 residents aged $\geq 65$ years and living in the designated area for $>5$ years participated in the baseline survey; 4,543 (86\%) completed the 2 -year follow-up. We excluded 1,402 participants with missing information regarding NC, WC, and weight or height at baseline or follow-up, and 495 with medical illnesses at baseline (cardiac, renal, or liver disease, AIDS, and other systemic diseases, e.g., cancer). Ultimately, 2,646 participants (1,288 men and 1,358 women) were included in the final analysis.

Each subject underwent a medical history and physical examination. Current smokers were defined as subjects who, at the time of interview, reported smoking every day or some days and had smoked at least 100 cigarettes during their lifetime. Alcohol use was defined as consumption of $>20-30 \mathrm{~g}$ and $>10-20 \mathrm{~g}$ of ethanol per day for men and women, respectively. A history of diabetes was defined as individuals with a history of glucose-lowering medication. A history of cardiovascular disease was defined as having had angiography-confirmed coronary stenosis with a lumen narrowing of $50 \%$, or acute myocardial infarction. A history of hypertension was defined as having had systolic blood pressure (SBP) $\geq 140$ $\mathrm{mm} \mathrm{Hg}$, and/or diastolic blood pressure (DBP) $\geq 90 \mathrm{~mm} \mathrm{Hg}$, or oral antihypertensive treatment.

\section{Anthropometry}

Blood pressure was measured using the standard method. The subjects wore light clothes without shoes for the measurement of body weight and height. BMI was calculated. NC was measured at the upper margin of the laryngeal prominence, with the head erect and the eyes facing forward. WC was measured at the level of the umbilicus.

\section{Diabetes Definition}

Diabetes was diagnosed based on the 1999 WHO criteria [17]. Given our lack of postprandial glucose detection, diabetes was diagnosed based only on fasting plasma glucose (FPG) values and diabetes medication history.

\section{Statistics Analyses}

All statistical analyses were performed using IBM SPSS Statistics v21.0 (IBM Corp., Armonk, NY, USA). Categorical data are described as percentages and continuous data as means and standard deviations (SD). Given that all continuous data were abnormally distributed, the Mann-Whitney U test was used to compare data between men and women. Pearson's correlation was used to analyze correlations between baseline $\mathrm{NC}$ and continuous variables. Binary logistic regression analysis was performed to explore diabetes-related risk factors in the baseline population. Risk was described using odds ratio (OR) and 95\% CI. The Cox regression model was used to analyze associations between baseline NC and new-onset type 2 diabetes in the following 2 years. Risk was described as hazard ratio (HR) and 95\% CI. Statistical significance was set at $p<0.05$.

\section{Results}

\section{Baseline Characteristics of the Study Participants}

Participants had a mean age of $71.0 \pm 5.8$ years at baseline. The main characteristics of the study population are summarized in Table 1 . The mean NC was $37.8 \pm 2.8 \mathrm{~cm}$ for men and $34.3 \pm 2.8 \mathrm{~cm}$ for women. Of the 2,646 participants, 219 developed new-onset diabetes at baseline (the prevalence of new diabetes diagnosis was $8.8 \%$ for men and $8.9 \%$ for women), while 98 had developed newonset diabetes at follow-up after 2 years.

\section{Correlation between NC and Anthropological and Metabolic Variables}

Correlations between NC and anthropological and metabolic variables are presented in Table 2. Accordingly, a weak negative correlation between $\mathrm{NC}$ and age was observed in men $(r=-0.09, p=0.001)$, but no correlation was noted in women $(p=0.427)$. Significant correlations between NC and weight, BMI, and WC were observed $(r=0.31,0.24,0.34$ in men and $r=0.29,0.27,0.28$ in women, respectively; all $p<0.001)$. Moreover, significant correlations between NC and FPG were found $(r=0.08$ in men and $r=0.14$ in women; all $p<0.05$ ). Significant correlations between NC and SBP and alanine aminotransferase (ALT) were observed in men (all $p<0.05)$, but a weak or no correlation was noted in women (all $p \geq$ 
Table 1. Baseline characteristics of participants according to sex

\begin{tabular}{lccc}
\hline Parameters & Males & Females & $p$ value* \\
\hline$N$ & 1,288 & 1,358 & - \\
Age, years & $70.9 \pm 5.4$ & $71.1 \pm 5.8$ & 0.386 \\
Current smoker & 12.8 & 0.5 & $<0.001$ \\
Alcohol use & 37.7 & 30.8 & 0.001 \\
Exercise & 49.5 & 50.5 & 0.141 \\
A history of diabetes & 15.1 & 17 & 0.185 \\
A history of cardiovascular disease & 15.8 & 20.3 & 0.014 \\
A history of hypertension & 46.1 & 50.9 & $<0.001$ \\
Weight, kg & $67.9 \pm 9.9$ & $58.9 \pm 9.4$ & 0.477 \\
BMI & $24.3 \pm 3.7$ & $24.4 \pm 3.8$ & $<0.001$ \\
WC, cm & $86.8 \pm 9.3$ & $84.6 \pm 9.8$ & 0.001 \\
NC, cm & $37.8 \pm 2.8$ & $34.3 \pm 2.8$ & 0.002 \\
SBP, mm Hg & $138.2 \pm 21.7$ & $141.0 \pm 22.5$ & $<009$ \\
DBP, mm Hg & $75.3 \pm 12.4$ & $74.1 \pm 12.4$ & 0.001 \\
TG, mmol/L & $1.4+0.9$ & $1.7+1.1$ & 0.242 \\
TC, mmol/L & $4.5+0.9$ & $5.0+0.9$ & 0.002 \\
FPG, mmol/L & $5.9 \pm 1.5$ & $5.8 \pm 1.5$ & 0.481 \\
ALT, IU/L & $20.7 \pm 14.5$ & $19.0 \pm 11.9$ & 8.9 \\
New-onset diabetes & 8.8 & 8.9 & \\
\hline
\end{tabular}

Values are expressed as percentages or means \pm standard deviation, unless otherwise indicated. ${ }^{*} p$ values $<$ 0.05 were considered significant. BMI, body mass index; DBP, diastolic blood pressure; FPG, fasting plasma glucose; NC, neck circumference; SBP, systolic blood pressure; TC, total cholesterol; TG, triglycerides; WC, waist circumference; ALT, alanine aminotransferase.

0.05). Furthermore, no significant correlations between $\mathrm{NC}$ and DBP, triglycerides (TG), and total cholesterol (TC) were noted in either gender ( $p>0.05$ for both).

\section{Association between NC and Diabetes Prevalence at Baseline}

Table 3 outlines the independent risk factors for type 2 diabetes prevalence using binary logistic regression analysis at baseline. Considering newly diagnosed diabetes at baseline as the independent variable, and age, exercise, current smoking, alcohol use, WC, NC, SBP, DBP, TG, TC, and ALT as the covariate variables, the binary logistic regression analysis showed that $\mathrm{NC}$ was an independent risk factor for type 2 diabetes prevalence in men (OR 1.18, 95\% CI 1.07-1.30, $p=0.001$ ) and women (OR $1.25,95 \%$ CI $1.13-1.38, p<0.001)$.

\section{Association between Baseline NC and the Incidence of} Diabetes at Follow-Up after 2 Years

To explore the association between baseline NC and the incidence of diabetes, multiple Cox regression was used to analyze independent risk factors for new-onset diabetes according to gender in the cohort population (Table 4). Considering new-onset diabetes as the independent variable, and age, exercise, current smoking, al-
Table 2. Correlation between neck circumference and anthropological and metabolic variables

\begin{tabular}{lcccccc}
\hline & \multicolumn{2}{c}{ Males } & & \multicolumn{2}{l}{ Females } \\
\cline { 2 - 3 } \cline { 6 - 7 } & $r$ & $p^{*}$ & & $r$ & \multicolumn{2}{c}{$p^{*}$} \\
\hline Age, years & -0.09 & 0.001 & & -0.02 & 0.427 \\
Weight, kg & 0.31 & $<0.001$ & & 0.29 & $<0.001$ \\
BMI & 0.24 & $<0.001$ & & 0.27 & $<0.001$ \\
WC, cm & 0.34 & $<0.001$ & & 0.28 & $<0.001$ \\
SBP, mm Hg & 0.07 & 0.02 & & 0.02 & 0.05 \\
DBP, mm Hg & 0.04 & 0.166 & & 0.04 & 0.199 \\
FPG, mmol/L & 0.08 & 0.007 & & 0.14 & $<0.001$ \\
TC, mmol/L & 0.02 & 0.583 & & 0.04 & 0.195 \\
TG, mmol/L & 0.05 & 0.069 & & 0.05 & 0.076 \\
ALT, IU/L & 0.09 & 0.001 & & 0.08 & 0.748 \\
\hline
\end{tabular}

* $p$ values $<0.05$ were considered significant. BMI, body mass index; DBP, diastolic blood pressure; FPG, fasting plasma glucose; SBP, systolic blood pressure; TC, total cholesterol; TG, triglycerides; WC, waist circumference; ALT, alanine aminotransferase.

cohol use, BMI, NC, WC, SBP, DBP, TG, TC, and ALT at baseline as the covariate variables, the Cox regression analysis revealed that $\mathrm{NC}$ was an independent risk factor for diabetes in men (HR 1.14, 95\% CI 1.05-1.23, $p=$ 0.002 ) and women (HR 1.18, 95\% CI 1.10-1.27, $p<0.001$ ). 
Table 3. Binary regression analysis of diabetes-related risk factors in baseline population by gender

\begin{tabular}{|c|c|c|c|c|}
\hline & \multicolumn{2}{|l|}{ Males } & \multicolumn{2}{|l|}{ Females } \\
\hline & OR (95\% CI) & $p$ value* & OR (95\% CI) & $p$ value* \\
\hline Age, years & $1.03(0.98,1.09)$ & 0.284 & $1.00(0.95,1.06)$ & 0.855 \\
\hline Current smoker (yes vs. no) & $0.96(0.42,2.20)$ & 0.919 & $3.95(0.32,49.1)$ & 0.285 \\
\hline Alcohol use (yes vs. no) & $1.09(0.515,2.30)$ & 0.826 & $3.12(0.70,14.0)$ & 0.136 \\
\hline Exercise (yes vs. no) & $0.45(0.14,1.45)$ & 0.182 & $0.52(0.17,1.64)$ & 0.269 \\
\hline BMI & $1.04(0.91,1.19)$ & 0.586 & $0.95(0.86,1.05)$ & 0.276 \\
\hline $\mathrm{WC}, \mathrm{cm}$ & $0.97(0.93,1.02)$ & 0.268 & $1.00(0.96,1.04)$ & 0.991 \\
\hline $\mathrm{NC}, \mathrm{cm}$ & $1.18(1.07,1.31)$ & 0.001 & $1.25(1.13,1.38)$ & 0.000 \\
\hline $\mathrm{SBP}, \mathrm{mm} \mathrm{Hg}$ & $0.99(0.97,1.01)$ & 0.457 & $1.00(0.99,1.02)$ & 0.617 \\
\hline $\mathrm{DBP}, \mathrm{mm} \mathrm{Hg}$ & $1.00(0.97,1.04)$ & 0.993 & $1.01(0.98,1.04)$ & 0.680 \\
\hline $\mathrm{TC}, \mathrm{mmol} / \mathrm{L}$ & $0.96(0.68,1.36)$ & 0.817 & $0.93(0.68,1.28)$ & 0.660 \\
\hline $\mathrm{TG}, \mathrm{mmol} / \mathrm{L}$ & $1.11(0.87,1.42)$ & 0.414 & $1.11(0.88,1.39)$ & 0.374 \\
\hline ALT, IU/L & $1.01(0.99,1.04)$ & 0.375 & $1.01(0.99,1.04)$ & 0.187 \\
\hline
\end{tabular}

* $p$ values < 0.05 were considered significant. BMI, body mass index; DBP, diastolic blood pressure; FPG, fasting plasma glucose; NC, neck circumference; SBP, systolic blood pressure; TC, total cholesterol; TG, triglycerides; WC, waist circumference; ALT, alanine aminotransferase; OR, odds ratio.

Table 4. Cox regression analysis of the association between baseline clinical characteristics and the risk of newonset diabetes after 2 years

\begin{tabular}{|c|c|c|c|c|}
\hline & \multicolumn{2}{|l|}{ Males } & \multicolumn{2}{|l|}{ Females } \\
\hline & HR (95\% CI) & $p$ value* & HR (95\% CI) & $p$ value* \\
\hline Age, years & $1.02(0.97,1.07)$ & 0.448 & $1.00(0.95,1.04)$ & 0.865 \\
\hline Current smoker (yes vs. no) & $0.95(0.49,1.86)$ & 0.889 & $2.82(0.37,21.50)$ & 0.318 \\
\hline Alcohol use (yes vs. no) & $1.13(0.63,2.05)$ & 0.677 & $1.86(0.55,6.21)$ & 0.316 \\
\hline Exercise (yes vs. no) & $0.57(0.22,1.45)$ & 0.236 & $0.74(0.27,2.02)$ & 0.551 \\
\hline BMI & $1.05(0.94,1.17)$ & 0.394 & $0.97(0.89,1.05)$ & 0.392 \\
\hline $\mathrm{WC}, \mathrm{cm}$ & $0.98(0.94,1.02)$ & 0.346 & $1.00(0.97,1.04)$ & 0.942 \\
\hline $\mathrm{NC}, \mathrm{cm}$ & $1.14(1.05,1.23)$ & 0.002 & $1.18(1.10,1.27)$ & 0.000 \\
\hline $\mathrm{SBP}, \mathrm{mm} \mathrm{Hg}$ & $1.00(0.98,1.02)$ & 0.970 & $1.00(0.99,1.02)$ & 0.683 \\
\hline $\mathrm{DBP}, \mathrm{mm} \mathrm{Hg}$ & $0.99(0.98,1.02)$ & 0.694 & $1.01(0.98,1.03)$ & 0.611 \\
\hline $\mathrm{TC}, \mathrm{mmol} / \mathrm{L}^{\circ}$ & $1.09(0.83,1.45)$ & 0.522 & $0.95(0.73,1.22)$ & 0.678 \\
\hline $\mathrm{TG}, \mathrm{mmol} / \mathrm{L}$ & $1.10(0.94,1.29)$ & 0.226 & $1.12(0.94,1.32)$ & 0.201 \\
\hline ALT, IU/L & $1.02(1.00,1.04)$ & 0.037 & $1.02(1.00,1.04)$ & 0.013 \\
\hline
\end{tabular}

* $p$ values < 0.05 were considered significant. BMI, body mass index; DBP, diastolic blood pressure; FPG, fasting plasma glucose; NC, neck circumference; SBP, systolic blood pressure; TC, total cholesterol; TG, triglyceride; WC, waist circumference; ALT, alanine aminotransferase; HR, hazard ratio.

\section{Discussion}

This cohort study explored the association between $\mathrm{NC}$ and the prevalence and incidence of diabetes in elderly individuals living in the community of the Pudong New District of Shanghai, China. Our cohort analysis indicated that NC could be used as alternative measure- ment for identifying the risk of developing type 2 diabetes in Chinese elderly individuals.

Throughout recent years, studies regarding the correlation between NC and FPG, impaired glucose regulation, or type 2 diabetes prevalence have gradually emerged [ 8 , 14, 15, 18-22]. The Framingham Heart Study showed that the risk of new diabetes (diabetes defined using FPG) 
increased 1.57-fold for each SD of NC after adjusting for $\mathrm{BMI}$ and WC, with this increase being more significant in women than in men [14]. In their 10-year longitudinal study conducted in Korea (KoGES study), Cho et al. [15] also found that $\mathrm{NC}$ was still an independent risk factor for new-onset diabetes after adjusting for other diabetes risk factors. Recent studies in the Chinese population have also found that NC was associated with cardiometabolic diseases. In fact, He et al. [21] found that NC was associated with the risk of developing gestational diabetes during early pregnancy. Meanwhile, a cross-sectional study in southeast China by Fu et al. [22] found that NC was associated with diabetes and other cardiometabolic diseases. Ting et al. [16] also revealed that NC exhibited a strong association with the occurrence of diabetes over a long period of time ( 8.87 years) in the Taiwanese population. However, there are insufficient data to support the association between $\mathrm{NC}$ and the risk of diabetes in elderly individuals who are already diabetic high-risk subjects. Our findings added new evidence about the elderly to support NC as an important risk factor for type 2 diabetes.

The underlying mechanism of the association between NC and risk of type 2 diabetes remains unclear. Nonetheless, the lipolytic activity of upper-body fat may be one mechanism explaining such an association. Research into regional lipolysis has demonstrated that upper-body subcutaneous fat accounts for a higher percentage of circulating free fatty acids (FFAs) than visceral fat, especially in obese subjects $[23,24]$. Obesity and elevated FFA levels are closely linked to insulin resistance and are a major risk factor for the development of diabetes $[25,26]$.

According to our study results, $\mathrm{NC}$ was independently associated with the occurrence of diabetes in Chinese elderly individuals. Some limitations merit discussion. Firstly, given the difficulty of conducting oral glucose tolerance tests in a large number of elderly individuals (the need for elderly individuals to take off their coat for venous blood collection, waiting time, etc.), only FPG and diabetes medication history was used to diagnose diabetes, which may have contributed to an underestimation of the incidence of diabetes in the study population. Secondly, our results may not be extrapolated to other racial or age groups, considering that the studied subjects were predominantly Chinese elderly individuals. Finally, the 2-year follow-up was relatively short for a longitudinal study. Future studies with longer follow-up duration are therefore needed to more clearly determine the impact of $\mathrm{NC}$ on the risk of developing diabetes.

\section{Conclusions}

We found that a larger NC was associated with a higher risk of developing type 2 diabetes in Chinese elderly individuals. Studies with larger sample sizes and longer follow-up durations are therefore essential to be able to clearly examine the relationship between $\mathrm{NC}$ and the risk of diabetes.

\section{Acknowledgements}

We are grateful to the participants, physicians, and nurses at the Department of Endocrinology, Shanghai East Hospital and Shanghai Puxing Community Health-Care Center for taking part in this study.

\section{Statement of Ethics}

All participants gave their written informed consent. This study was approved by the institutional review board of Shanghai East Hospital (No. 2013009) and conducted in accordance with the Declaration of Helsinki.

\section{Conflict of Interest Statement}

The authors declare that they have no competing interests.

\section{Funding Sources}

The testing costs of all participants in this study were funded by Key Specialty Construction Project of Pudong Health and Family Planning Commission of Shanghai (grant No. PWZz2017-12). The study was also funded by a Special Fund for Health Science and Technology of Livelihood Research of Shanghai Pudong New Area (PKJ2017-Y63) for payment of service charges and information engineering costs.

\section{Author Contributions}

B.F. designed the study and oversaw the project implementation, and participated in data analyses and interpretation, and the writing of the paper. D.S., X.L., Z.Q., and H.L. conceived and carried out experiments. Q.Y. and D.S. participated in data collection, analysis, and interpretation, and the writing of the paper. All authors were involved in writing the paper and gave final approval of the submitted and published versions. 


\section{References}

1 Sjöström CD, Håkangård AC, Lissner L, Sjöström L. Body compartment and subcutaneous adipose tissue distribution - risk factor patterns in obese subjects. Obes Res. 1995 Jan;3(1):9-22.

2 Ben-Noun L, Sohar E, Laor A. Neck circumference as a simple screening measure for identifying overweight and obese patients. Obes Res. 2001 Aug;9(8):470-7.

3 Onat A, Hergenç G, Yüksel H, Can G, Ayhan E, Kaya Z, et al. Neck circumference as a measure of central obesity: associations with metabolic syndrome and obstructive sleep apnea syndrome beyond waist circumference. Clin Nutr. 2009 Feb;28(1):46-51.

4 Yang GR, Yuan SY, Fu HJ, Wan G, Zhu LX, $\mathrm{Bu}$ XL, et al.; Beijing Community Diabetes Study Group. Neck circumference positively related with central obesity, overweight, and metabolic syndrome in Chinese subjects with type 2 diabetes: Beijing Community Diabetes Study4.Diabetes Care.2010Nov;33(11):2465-

5 Nafiu OO, Burke C, Lee J, Voepel-Lewis T, Malviya S, Tremper KK. Neck circumference as a screening measure for identifying children with high body mass index. Pediatrics. 2010 Aug;126(2):e306-10.

6 Hatipoglu N, Mazicioglu MM, Kurtoglu S, Kendirci M. Neck circumference: an additional tool of screening overweight and obesity in childhood. Eur J Pediatr. 2010 Jun;169(6):733-9.

7 Mazicioglu MM, Kurtoglu S, Ozturk A, Hatipoglu N, Cicek B, Ustunbas HB. Percentiles and mean values for neck circumference in Turkish children aged 6-18 years. Acta Paediatr. 2010 Dec;99(12):1847-53.

8 Androutsos O, Grammatikaki E, Moschonis G, Roma-Giannikou E, Chrousos GP, Manios $Y$, et al. Neck circumference: a useful screening tool of cardiovascular risk in children. Pediatr Obes. 2012 Jun;7(3):187-95.

9 Lou DH, Yin FZ, Wang R, Ma CM, Liu XL, Lu Q. Neck circumference is an accurate and simple index for evaluating overweight and obesity in Han children. Ann Hum Biol. 2012 Mar;39(2):161-5.

10 Ma C, Wang R, Liu Y, Lu Q, Liu X, Yin F. Diagnostic performance of neck circumference to identify overweight and obesity as defined by body mass index in children and adolescents: systematic review and meta-analysis. Ann Hum Biol. 2017 May;44(3):223-9.

11 Li HX, Zhang F, Zhao D, Xin Z, Guo SQ, Wang SM, et al. Neck circumference as a measure of neck fat and abdominal visceral fat in Chinese adults. BMC Public Health. 2014 Apr;14(1):311.

12 Xu Y, Jian C, Ma X, Shen Y, Wang Y, Bao Y. Comparison of neck and waist circumferences for identifying subclinical atherosclerosis in a community-based population. Diabetes Metab Res Rev. 2020. DOI: 10.1002/ dmrr.3382.

13 Yan Q, Sun D, Li X, Zheng Q, Li L, Gu C, et al. Neck circumference is a valuable tool for identifying metabolic syndrome and obesity in Chinese elder subjects: a community-based study. Diabetes Metab Res Rev. 2014 Jan;30(1):69-76.

14 Preis SR, Pencina MJ, D’Agostino RB Sr, Meigs JB, Vasan RS, Fox CS. Neck circumference and the development of cardiovascular disease risk factors in the Framingham Heart Study. Diabetes Care. 2013 Jan;36(1):e3.

15 Cho NH, Oh TJ, Kim KM, Choi SH, Lee JH, Park KS, et al. Neck Circumference and Incidence of Diabetes Mellitus over 10 Years in the Korean Genome and Epidemiology Study (KoGES). Sci Rep. 2015 Dec;5(1):18565.

16 Ting MK, Liao PJ, Wu IW, Chen SW, Yang NI, Lin TY, et al. Predicting Type 2 Diabetes Mellitus Occurrence Using Three-Dimensional Anthropometric Body Surface Scanning Measurements: A Prospective Cohort Study. J Diabetes Res. 2018 Jul;2018:6742384.

17 Alberti KG, Zimmet PZ. Definition, diagnosis and classification of diabetes mellitus and its complications. Part 1: diagnosis and classifi- cation of diabetes mellitus provisional report of a WHO consultation. Diabet Med. 1998 Jul;15(7):539-53.

18 Hoebel S, Malan L, de Ridder JH. Determining cut-off values for neck circumference as a measure of the metabolic syndrome amongst a South African cohort: the SABPA study. Endocrine. 2012 Oct;42(2):335-42.

19 Stabe C, Vasques AC, Lima MM, Tambascia MA, Pareja JC, Yamanaka A, et al. Neck circumference as a simple tool for identifying the metabolic syndrome and insulin resistance: results from the Brazilian Metabolic Syndrome Study. Clin Endocrinol (Oxf). 2013 Jun;78(6):874-81.

20 Khalangot M,Gurianov V,Okhrimenko N, Luzanchuk I, Kravchenko V. Neck circumference as a risk factor of screen-detected diabetes mellitus: community-based study. Diabetol Metab Syndr. 2016 Feb;8:12.

$21 \mathrm{He} \mathrm{F,} \mathrm{He} \mathrm{H,} \mathrm{Liu} \mathrm{W,} \mathrm{Lin} \mathrm{J,} \mathrm{Chen} \mathrm{B,} \mathrm{Lin} \mathrm{Y,} \mathrm{et} \mathrm{al.}$ Neck circumference might predict gestational diabetes mellitus in Han Chinese women: A nested case-control study. J Diabetes Investig. 2017 Mar;8(2):168-73.

$22 \mathrm{Fu} \mathrm{W,} \mathrm{Zou} \mathrm{L,} \mathrm{Yin} \mathrm{X,} \mathrm{Wu} \mathrm{J,} \mathrm{Zhang} \mathrm{S,} \mathrm{Mao} \mathrm{J,} \mathrm{et}$ al. Association between neck circumference and cardiometabolic disease in Chinese adults: a community-based cross-sectional study. BMJ Open. 2019 Dec;9(12):e026253.

23 Guo Z, Hensrud DD, Johnson CM, Jensen MD. Regional postprandial fatty acid metabolism in different obesity phenotypes. Diabetes. 1999 Aug;48(8):1586-92.

24 Jensen MD. Is Visceral Fat Involved in the Pathogenesis of the Metabolic Syndrome? Human Model. Obesity (Silver Spring). 2006 Feb; 14 Suppl 1:20S-24S.

25 Boden G, Shulman GI. Free fatty acids in obesity and type 2 diabetes: defining their role in the development of insulin resistance and beta-cell dysfunction. Eur J Clin Invest. 2002 Jun;32 Suppl 3:14-23.

26 Yazıcı D, Sezer H. Insulin Resistance, Obesity and Lipotoxicity. Adv Exp Med Biol. 2017;960:277-304. 